\title{
Filters in algebras of fuzzy logics
}

\author{
Martin Víta and Petr Cintula \\ Institute of Computer Science, Academy of Sciences of the Czech Republic \\ Pod Vodárenskou věží 2, 182 07, Prague, Czech Republic. \{vita,cintula\}@cs.cas.cz
}

\begin{abstract}
This paper presents a generalization of many particular results about special types of filters (e.g., (positive) implicative, fantastic) on algebras of nonclassical (mostly fuzzy) logics. Our approach is rooted in the framework of Abstract Algebraic Logic, and is based on the close connection between the filter-defining conditions and alternative axiomatizations of the logics involved. We identify four main kinds of theorems proved in the literature and we formulate general theorems which (provided a simple syntactical proof) yield the majority of published results as their direct consequences.
\end{abstract}

Keywords: Filters, Abstract Algebraic Logic, Rasiowa-Implicative Logics

\section{Introduction}

The study of special types of filters on algebras of certain non-classical logics has become very popular in the last years. Many journal papers, e.g. Haveshki ([7]), Šalounová and Rachůnek ([10]), Kondo and Dudek [9] and others contain (among others) four main types of results which we are going to demonstrate on the following particular examples (see Haveskhi [7], Kondo and Dudek [9]).

We assume that the reader is familiar with the notion of BL-algebra [6], we recall that a (BL-)filter (a set $F$ containing $\overline{1}$ such that $y \in F$ whenever $x, x \rightarrow y \in F)$ is fantastic if $z \rightarrow(y \rightarrow x) \in F$ and $z \in F$ imply $((x \rightarrow y) \rightarrow y) \rightarrow x \in F$. Then for each BL-algebra $\boldsymbol{A}$ holds:

1. The following statements are equivalent:

- Every filter on $\boldsymbol{A}$ is fantastic.

- $\{\overline{1}\}$ is a fantastic filter on $\boldsymbol{A}$.

- $\boldsymbol{A}$ is an MV-algebra.

2. A filter $F$ is fantastic iff for each $x \in A$ holds: $((x \rightarrow \overline{0}) \rightarrow \overline{0}) \rightarrow x \in F$.

3. If $F$ is fantastic, then so is any filter $G \supseteq F$.

4. A filter $F$ is fantastic iff every filter on the quotient algebra $\boldsymbol{A} / F$ is a fantastic filter.

The primary purpose of this contribution is to show triviality of these results once they are formulated in a proper general framework, in this case the one of Abstract Algebraic Logic (AAL). We develop a very simple abstract theory covering (among others) all the results of the four presented types. In particular, we reduce all these theorems to a simple, purely syntactical, problem of alternative axiomatization of some particular logics.

The secondary but equally important goal of this paper is to demonstrate that Abstract Algebraic Logic provides powerful tools for studying algebras of non-classical logics and should be always taken into account before particular results on particular algebras are proved.

\section{Preliminaries}

\subsection{Prominent logics}

We use several prominent non-classical logics to provide examples of our claims. The weakest logic we will consider here is called Full Lambek logic with exchange and weakening [5] (also known as Höhle's Monoidal Logic [8]). It has four primitive binary connectives $\rightarrow, \&, \wedge, \vee$, and the truth constant $\overline{0}$ (and defined connectives $\neg \varphi=\varphi \rightarrow \overline{0}$ and $\overline{1}=\overline{0} \rightarrow \overline{0})$; and it can be axiomatized with modus ponens (from $\varphi$ and $\varphi \rightarrow \psi$ infer $\psi$ ) as the only deduction rule and the axioms listed in Table 1. Other prominent logics considered in this paper are listed in Table 2.

Table 1: Axiomatic system of $\mathrm{FL}_{e w}$

$\begin{array}{ll}(\mathrm{Ax} 1) & (\varphi \rightarrow \psi) \rightarrow((\psi \rightarrow \chi) \rightarrow(\varphi \rightarrow \chi)) \\ (\mathrm{Ax} 2) & (\varphi \& \psi) \rightarrow \varphi \\ (\mathrm{Ax} 3) & (\varphi \& \psi) \rightarrow(\psi \& \varphi) \\ (\mathrm{Ax} 4 \mathrm{a}) & \varphi \wedge \psi \rightarrow \varphi \\ (\mathrm{Ax} 4 \mathrm{~b}) & \varphi \wedge \psi \rightarrow \psi \wedge \varphi \\ (\mathrm{Ax} 4 \mathrm{c}) & (\chi \rightarrow \varphi) \rightarrow((\chi \rightarrow \psi) \rightarrow(\chi \rightarrow \varphi \wedge \psi)) \\ (\mathrm{Ax} 4 \mathrm{~d}) & \varphi \rightarrow \varphi \vee \psi \\ (\mathrm{Ax} 4 \mathrm{e}) & \varphi \vee \psi \rightarrow \psi \vee \varphi \\ (\mathrm{Ax} 4 \mathrm{f}) & (\varphi \rightarrow \chi) \rightarrow((\psi \rightarrow \chi) \rightarrow(\varphi \vee \psi \rightarrow \chi)) \\ (\mathrm{Ax} 5 \mathrm{a}) & (\varphi \rightarrow(\psi \rightarrow \chi)) \rightarrow(\varphi \& \psi \rightarrow \chi) \\ (\mathrm{Ax} 5 \mathrm{~b}) & (\varphi \& \psi \rightarrow \chi)) \rightarrow(\varphi \rightarrow(\psi \rightarrow \chi)) \\ (\mathrm{Ax} 6) & \overline{0} \rightarrow \varphi\end{array}$

Table 2: Prominent logics

\begin{tabular}{|c|c|}
\hline Logic & definition \\
\hline Intuitionistic & $\mathrm{FL}_{e w}+\varphi \rightarrow \varphi \& \varphi$ \\
\hline $\operatorname{divFL}_{e w}$ & $\mathrm{FL}_{e w}+\varphi \wedge \psi \rightarrow \varphi \&(\varphi \rightarrow \psi)$ \\
\hline Classical & $\mathrm{FL}_{e w}+\varphi \vee \neg \varphi$ \\
\hline MTL & $\mathrm{FL}_{e w}+(\varphi \rightarrow \psi) \vee(\psi \rightarrow \varphi)$ \\
\hline IMTL & MTL $+\neg \neg \varphi \rightarrow \varphi$ \\
\hline $\mathrm{BL}$ & MTL $+\varphi \wedge \psi \rightarrow \varphi \&(\varphi \rightarrow \psi)$ \\
\hline Łukasiewicz & $\mathrm{BL}+\neg \neg \varphi \rightarrow \varphi$ \\
\hline Gödel & $\mathrm{BL}+\varphi \rightarrow \varphi \& \varphi$ \\
\hline
\end{tabular}




\subsection{Rasiowa-implicative logic}

This subsection includes basic definitions and it also establishes notational conventions. For comprehensive introduction to Abstract Algebraic Logic see $[4,3]$. We assume that the notion of propositional language $\mathcal{L}$ is defined as usual. We denote by the symbol $\boldsymbol{F} \boldsymbol{m}_{\mathcal{L}}$ the free term algebra with signature $\mathcal{L}$ over a denumerable set of generators (in this context called the propositional variables). The universe of this free term algebra will be denoted by $F m_{\mathcal{L}}$ (and the same convention we will use in the whole text: if $\boldsymbol{A}$ is an algebra, then $A$ is its universe). The elements of $F m_{\mathcal{L}}$ are called formulas. Somewhere in the text we omit the symbol of the language when it is clear from the context.

The endomorphisms of the algebra $\boldsymbol{F} \boldsymbol{m}_{\mathcal{L}}$ are called $\mathcal{L}$-substitutions and play an important role when defining a notion of logic.

Definition 1 An $\mathcal{L}$-consecution is a pair $\langle\Gamma, \varphi\rangle$, usually written as $\Gamma \triangleright \varphi$, where $\Gamma \cup\{\varphi\} \subseteq F m_{\mathcal{L}}$. $A$ consecution $\Gamma \triangleright \varphi$ is finitary if $\Gamma$ is finite.

We often identify a consecution of the form $\emptyset \triangleright \varphi$ with the formula $\varphi$ itself. Clearly, a set of consecutions L can be viewed as a relation between sets of formulas and single formulas, we write $\Gamma \vdash_{L} \varphi$ instead of $\Gamma \triangleright \varphi \in \mathrm{L}$.

Definition 2 A finitary propositional logic L, a logic for short, in the language $\mathcal{L}$ is a set of $\mathcal{L}$ consecutions such that:

1. $\Gamma, \varphi \vdash_{\mathrm{L}} \varphi$,

2. if $\Gamma \vdash_{\mathrm{L}} \varphi$ and for every $\psi \in \Gamma, \Delta \vdash_{\mathrm{L}} \psi$, then $\Delta \vdash_{\mathrm{L}} \varphi$

3. $\Gamma \vdash_{\mathrm{L}} \varphi$ implies $\sigma[\Gamma] \vdash_{\mathrm{L}} \sigma(\varphi)$ for every $\mathcal{L}$-substitution $\sigma$.

4. if $\Gamma \vdash_{\mathrm{L}} \varphi$ then there is a finite set $\Delta \subseteq \Gamma$ such that $\Delta \vdash_{\mathrm{L}} \varphi$.

The first two conditions imply that $\vdash_{\mathrm{L}}$ is a consequence relation in the sense of Tarski, the third one is known as structurality or substitution invariance, the final one is called finitarity. We omit the subscript L when clear from the context.

A logic can be syntactically presented by means of several kinds of proof systems, in this contribution we always use Hilbert style calculi.

Definition 3 Let $\mathrm{L}$ be a logic. The set $\mathcal{A X}$ of finitary $\mathcal{L}$-consecutions is called an axiomatic system (or a presentation) of $\mathrm{L}$ if the relation $\vdash_{\mathrm{L}}$ coincides with the provability relation given by $\mathcal{A X}$ as a Hilbert style calculi: ${ }^{1}$ i.e., $\Gamma \vdash_{\mathrm{L}} \varphi$ if and only if there is a sequence of formulas $\left\langle\psi_{0}, \psi_{1}, \ldots, \psi_{n}\right\rangle$ such that $\psi_{n}=\varphi$ and for every $i<n, \psi_{i} \in \Gamma$ or it is an axiom or it follows from the previous elements of the proof by application of some of the deduction rules (formally: there is $\Delta \triangleright \alpha \in \mathcal{A X}$ and a substitution $\sigma$ such that $\sigma(\alpha)=\psi_{i}$ and $\left.\sigma[\Delta] \subseteq\left\{\psi_{0}, \ldots, \psi_{i-1}\right\}\right)$.

\footnotetext{
${ }^{1}$ Elements of $\mathcal{A X}$ of the form $\emptyset \triangleright \varphi$ are called axioms, the remaining ones are called deduction rules.
}

Definition 4 Let $\mathrm{L}$ be a logic and $R$ a set of consecutions. The weakest logic containing $\mathrm{L}$ and $R$ is denoted as $\mathrm{L}+R$.

Clearly, $\mathrm{L}+R$ is axiomatized by any of the presentations of $\mathrm{L}$ plus consecutions from $R$.

Definition 5 A logic $\mathrm{L}^{\prime}$ is an (axiomatic) extension of $\mathrm{L}$ if there is a set $R$ of consecutions (formulas) such that $\mathrm{L}^{\prime}=\mathrm{L}+R$.

All logics we consider in this paper are examples of Rasiowa-implicative logics (introduced in [11], the term 'Rasiowa-implicative' is from [2]). To simplify the exposition, we provide a slightly altered definition. We assume that the truth constant $\overline{1}$ is present in the language as a primitive connective rather than a derived one (by setting $\overline{1}=p \rightarrow p$ ).

Definition 6 A logic $\mathrm{L}$ in the language $\mathcal{L}$ is said to be Rasiowa-implicative logic if $\mathcal{L}$ contains a truth constant $\overline{1}$ and a binary connective $\rightarrow$ such that:



Let us introduce useful abbreviations: $\varphi \leftrightarrow \psi=$ $\{\varphi \rightarrow \psi, \psi \rightarrow \varphi\}$ and $\Gamma \vdash \Delta$ for $\Gamma \vdash \varphi$ for each $\varphi \in \Delta$. Let us stress that $\leftrightarrow$ is not a new connective but a set of formulae (in the literature $\leftrightarrow$ is called generalized equivalence).

\subsection{Algebras and filters}

We recall the basics of semantics of Rasiowaimplicative logics. Again, to streamline the presentation we do not define the usual notions in the canonical way, but we prefer more a didactic equivalent (in our context!) approach. Let us fix a Rasiowa-implicative logic in the language $\mathcal{L}$, by $\mathcal{L}$-algebra we understand algebra with signature $\mathcal{L}$ (e.g., each $\mathcal{L}$-algebra has a binary operation $\rightarrow$ and constant 1). Homomorphisms from $\boldsymbol{F} \boldsymbol{m}_{\mathcal{L}}$ to an $\mathcal{L}$ algebra $\boldsymbol{A}$ are called $\boldsymbol{A}$-evaluations.

Definition 7 An $\mathcal{L}$-algebra $\boldsymbol{A}$ is L-algebra, in symbols: $\boldsymbol{A} \in \mathbf{A L G}^{*}(\mathrm{~L})$, if

1. $\Gamma \vdash_{\mathrm{L}} \varphi$ implies that for each $\boldsymbol{A}$-evaluation e we have $e(\varphi)=\overline{1}$ whenever $e[\Gamma] \subseteq\{\overline{1}\}$,

2. $x \rightarrow y=\overline{1}$ and $y \rightarrow x=\overline{1}$ implies $x=y$,

for each $\Gamma \cup\{\varphi\} \subseteq \operatorname{Fm}_{\mathcal{L}}$ and $x, y \in A$.

Table 3 gives the list of prominent Rasiowa-implicative logics and indicates the traditional names for the corresponding classes $\mathbf{A L G}{ }^{*}(\mathrm{~L})$. Of course 
Table 3: Prominent Rasiowa-implicative logics

\begin{tabular}{|l|l|}
\hline Logic L & Traditional name of $\mathbf{A L G}^{*}(\mathrm{~L})$ \\
\hline $\mathrm{FL}_{e w}$ & $\begin{array}{l}\text { bounded commutative integral } \\
\text { residuated lattices }\end{array}$ \\
\hline Intuitionistic & Heyting algebras \\
\hline divFL $_{e w}$ & bounded commutative R $\ell$-monoids \\
\hline Classical & Boolean algebras \\
\hline MTL & MTL-algebras \\
\hline IMTL & MTL-algebras \\
\hline BL & BL-algebras \\
\hline Łukasiewicz & MV-algebras \\
\hline Gödel & Gödel algebras \\
\hline
\end{tabular}

those classes of algebras are defined in some explicit way (usually by equations), so it takes some work to show they coincide with our implicit definition.

It is well known that $\mathbf{A L G} \mathbf{L G}^{*}(\mathrm{~L})$ is a quasivariety (all classes of algebras mentioned in 3 are in fact varieties) and it is the equivalent algebraic semantics of $\mathrm{L}$ in the sense of Blok and Pigozzi [1], i.e.,

1. $\Gamma \vdash \varphi$ iff $\{\psi \approx \overline{1} \mid \psi \in \Gamma\} \models{ }_{\mathbf{A L G}^{*}(\mathrm{~L})} \varphi \approx \overline{1}$

2. $E \models \mathbf{A L G}^{*}(\mathrm{~L}) \varphi \approx \psi$ iff

$$
\{\alpha \leftrightarrow \beta \mid \alpha \approx \beta \in E\} \vdash_{\mathrm{L}} \varphi \leftrightarrow \psi
$$

3. $\overline{1} \leftrightarrow \varphi \vdash_{\mathrm{L}} \varphi$ and $\varphi \vdash_{\mathrm{L}} \overline{1} \leftrightarrow \varphi$

4. $\{\overline{1} \approx \varphi \rightarrow \psi, \overline{1} \approx \psi \rightarrow \varphi\} \models \mathrm{ALG}^{*}(\mathrm{~L}) \varphi \approx \psi$ and $\varphi \approx \psi \models_{\mathbf{A L G}^{*}(\mathrm{~L})}\{\overline{1} \approx \varphi \rightarrow \psi, \overline{1} \approx \psi \rightarrow \varphi\}$

Condition 1 is in fact the completeness theorem: $\Gamma \vdash_{\mathrm{L}} \boldsymbol{\varphi}$ iff for each $\boldsymbol{A} \in \mathbf{A L G}^{*}(\mathrm{~L})$ and each $\boldsymbol{A}$ evaluation $e$ we have $e(\varphi)=\overline{1}$ whenever $e[\Gamma] \subseteq\{\overline{1}\}$.

Filters corresponding to a logic $\mathrm{L}$ are, in Algebraic Logic, defined as sets closed under all possible derivations in the logic.

Definition 8 Let $\boldsymbol{A}$ be an $\mathcal{L}$-algebra. We say that $F \subseteq A$ is an L-filter, if for each $\Gamma \cup\{\varphi\} \subseteq \mathrm{Fm}_{\mathcal{L}}$ : $\Gamma \vdash_{\mathrm{L}} \varphi$ implies that for each $\boldsymbol{A}$-evaluation $e$, we have $e(\varphi) \in F$ whenever $e[\Gamma] \subseteq F$.

Note that we have defined the notion of L-filter on all algebras of the proper signature and not only on the L-algebras. This is related to a more general notion of semantics, the so-called logical matrices. Indeed $F$ is an L-filter in $\boldsymbol{A}$ iff the tuple $\langle\boldsymbol{A}, F\rangle$ is a matrix model of L. However to keep the exposition straightforward we will not go into details here.

It is well-known that the filters defined this way correspond straightforwardly to the congruences. Again, we present just a simplified version, in particular we will assume that $\mathbf{A} \mathbf{L G}^{*}(\mathrm{~L})$ is a variety. Then for any $\boldsymbol{A} \in \mathbf{A} \mathbf{L} \mathbf{G}^{*}(\mathrm{~A})$ holds:

- the relation $\sim_{F}$ defined as

$$
x \sim_{F} y \quad \text { iff } \quad x \rightarrow y \in F \text { and } y \rightarrow x \in F
$$

is a congruence on $\boldsymbol{A}$ for any L-filter $F$.

- the set $F_{\sim}=\{x \in A \mid x \sim \overline{1}\}$ is an L-filter for any congruence $\sim$ on $\boldsymbol{A}$.

- $F_{\sim_{F}}=F$ and $\sim_{F_{\sim}}=\sim$.

\footnotetext{
${ }^{2}$ In this paper, for simplicity, we will omit the prefixes and
} speak simply about 'residuated lattices' and 'R $\ell$-monoids'.
Thus we can reformulate our definition of $\mathbf{A L G}^{*}(\mathrm{~L})$ as: $\boldsymbol{A} \in \mathbf{A} \mathbf{L} \mathbf{G}^{*}(\mathrm{~L})$ iff $\{\overline{1}\}$ is an L-filter and $\sim_{\{\overline{1}\}}=\mathrm{Id}$.

The following observation greatly simplifies the task of recognizing whether $F$ is an L-filter (the first part is easily proved by induction over the complexity of the proof of $\varphi$ in $\Gamma$, the second one follows from the fact that $\overline{1}$ has to be in all filters and all axioms are necessarily evaluated to $\overline{1}$ in any L-algebra).

Lemma 9 Let $\mathcal{A X}$ be a presentation of a Rasiowaimplicative logic $\mathrm{L}$ and $\boldsymbol{A}$ an $\mathcal{L}$-algebra. Then $F \subseteq$ $A$ is L-filter iff

1. for each axiom $\varphi$ and for each $\boldsymbol{A}$-evaluation $e$ we have $e(\varphi) \in F$,

2. for each deduction rule $\Gamma \triangleright \varphi \in \mathcal{A} \mathcal{X}$ and for each $\boldsymbol{A}$-evaluation e we have $e(\varphi) \in F$ whenever $e[\Gamma] \subseteq F$.

If furthermore $\boldsymbol{A} \in \mathbf{A} \mathbf{L G}^{*}(\mathrm{~L})$ we can replace the first condition by

1.' $\overline{1} \in F$.

Because the logic BL (and all other logics mentioned in Table 2) can be presented with modus ponens $(\{p, p \rightarrow q\} \triangleright q)$ as the only deduction rule we obtain:

Example 10 Let $\boldsymbol{A}$ be a BL-algebra. Then $F \subseteq A$ is a BL-filter iff $\overline{1} \in F$ and for each $x, y \in A$ we have $y \in F$ whenever $x, x \rightarrow y \in F$.

\section{R-L-filters}

In this section we will present general results covering the four types mentioned in the introduction. Let us fix a Rasiowa-implicative logic $L$ in that language $\mathcal{L}$ and a set $R$ of $\mathcal{L}$-consecutions.

\subsection{Basic definition and examples}

Definition 11 Let $\boldsymbol{A} \in \mathbf{A L G}^{*}(\mathrm{~L})$. An L-filter $F$ is called $R$-L-filter if it satisfies the following condition for every $\mathbf{A}$-evaluation $e$ and every $\Gamma \triangleright \varphi \in R$ : if $e[\Gamma] \subseteq F$ then $e(\varphi) \in F$.

It is quite obvious that this general definition corresponds to the usual practice (in the papers mentioned in the introduction) of introducing a special classes of filters. $^{3}$ Indeed e.g. the fantastic BL-filters are $\{r \rightarrow(q \rightarrow p), r \triangleright((p \rightarrow q) \rightarrow q) \rightarrow p\}$-BL-filters in the sense of this definition; for more examples of this kind see Tables 4 and 5 .

\footnotetext{
${ }^{3}$ There is a minor difference, caused by the fact that the authors sometimes define, e.g. in BL, 'fantastic filter' as a set containing $\overline{1}$ and satisfying the 'fantastic' property. Only then (!) they prove that it is indeed a filter, i.e., closed under modus ponens. Thus strictly speaking our general notions coincide with theirs only after we prove that the particular properties in question implies that the set in question is indeed a filter.
} 
Table 4: Traditional formulation

\begin{tabular}{|l|l|}
\hline $\begin{array}{l}\text { Type of } \\
\text { L-filter }\end{array}$ & Condition in traditional form \\
\hline \hline Implicative & $\begin{array}{l}\text { if } x \rightarrow(y \rightarrow z) \in F \text { and } x \rightarrow y \in F, \\
\text { then } x \rightarrow z \in F\end{array}$ \\
\hline $\begin{array}{l}\text { Positive } \\
\text { implicative }\end{array}$ & $x \in F$, then $y \in F$ \\
\hline Fantastic & $\begin{array}{l}\text { if } z \rightarrow(y \rightarrow x) \in F \text { and } z \in F, \\
\text { then }((x \rightarrow y) \rightarrow y) \rightarrow x \in F\end{array}$ \\
\hline Boolean & $x \vee \neg x \in F$ \\
\hline Regular & $\neg \neg x \rightarrow x \in F$ \\
\hline
\end{tabular}

Table 5: Our formulation

\begin{tabular}{|l|l|}
\hline $\begin{array}{l}\text { Type of } \\
\text { L-filter }\end{array}$ & The corresponding set R \\
\hline \hline Implicative & $p \rightarrow(q \rightarrow r), p \rightarrow q \triangleright p \rightarrow r$ \\
\hline $\begin{array}{l}\text { Positive } \\
\text { implicative }\end{array}$ & $p \rightarrow((q \rightarrow r) \rightarrow q), p \triangleright q$ \\
\hline Fantastic & $r \rightarrow(q \rightarrow p), r \triangleright((p \rightarrow q) \rightarrow q) \rightarrow p$ \\
\hline Boolean & $\triangleright p \vee \neg p$ \\
\hline Regular & $\triangleright \neg \neg p \rightarrow p$ \\
\hline
\end{tabular}

\subsection{General results}

The main idea which allows us to summarize in this short subsection the results of dozens of articles about special types of filters can be roughly expressed as: "a special type of filter in an algebra of a weaker logic is a (standard) filter in a stronger logic". Recall that the expression $\mathrm{L}+R$ denotes the extension of the logic $\mathrm{L}$ by the consecutions $R$. Using Lemma 9 we can easily prove that:

Proposition 12 Let $\left.\boldsymbol{A} \in \mathbf{A L G}^{*}(\mathrm{~L})\right)$ and $F$ be an L-filter on $\boldsymbol{A}$. Then $F$ is an $R$-L-filter on $\boldsymbol{A}$ if and only if $F$ is $(\mathrm{L}+R)$-filter on $\boldsymbol{A}$.

This proposition together with the fact that the condition 1 in the definition of L-algebra could be replaced by ' $\{\overline{1}\}$ is L-filter' gives us:

Proposition 13 Let $R$ be a set of $\mathcal{L}$-consecutions and $\boldsymbol{A} \in \mathbf{A} \mathbf{L} \mathbf{G}^{*}(\mathrm{~L})$. Then $\boldsymbol{A} \in \mathbf{A} \mathbf{L} \mathbf{G}^{*}(\mathrm{~L}+R)$ iff $\{\overline{1}\}$ is $R$-L-filter.

Now we can present the first of four theorems generalizing the theorems known from the literature as presented in the introduction. Its proof is based on the previous proposition and the fact that for any logic L, any of its axiomatic extensions $\mathrm{L}^{\prime}$ and $\boldsymbol{A} \in \mathbf{A L G}^{*}\left(\mathrm{~L}^{\prime}\right)$ holds that $F$ is an L-filter iff it is an $\mathrm{L}^{\prime}$-filter (this fact easily follows from Lemma 9).

Theorem 14 (Results of type 1.) Let $R$ be $a$ set of $\mathcal{L}$-consecutions such that $\mathrm{L}+R$ is an axiomatic extension of $\mathrm{L}$. Then the following statements are equivalent for each $\boldsymbol{A} \in \mathbf{A} \mathbf{L} \mathbf{G}^{*}(\mathrm{~L})$ :

1. all $\mathrm{L}$-filters are $R$-L-filters,

2. $\{\overline{1}\}$ is $R$-L-filter,

3. $\boldsymbol{A} \in \mathbf{A L G}^{*}(\mathrm{~L}+R)$.
We can easily show that Łukasiewicz logic equals to $\mathrm{BL}+\{r \rightarrow(q \rightarrow p), r \triangleright((p \rightarrow q) \rightarrow q) \rightarrow p\}$ and so we obtain the first result mentioned in the introduction.

To prove the results of the second type we first observe that if $\mathrm{L}+R_{1} \subseteq \mathrm{L}+R_{2}$ then we can without a loss of generality assume that $R_{1} \subseteq R_{2}$. Thus if $F$ is an $R_{2}$-L-filter on $\boldsymbol{A}$, then $F$ is also an $R_{1}$-L-filter on $\boldsymbol{A}$. This gives us one non-trivial implication in the next theorem (1. implies 2.), the other one (3. implies 1.) is a rather straightforward consequence of the completeness theorem and Proposition 13.

Theorem 15 (Results of type 2.) Let L be $R_{1}$, $R_{2}$ be sets of $\mathcal{L}$-consecutions. Then the following statements are equivalent:

1. $\mathrm{L}+R_{1}=\mathrm{L}+R_{2}$.

2. For every $\boldsymbol{A} \in \mathbf{A L G}^{*}(\mathrm{~L})$ and $\mathrm{L}$-filter $F$ holds: $F$ is an $R_{1}$-L-filter iff $F$ is $R_{2}$-L-filter.

3. For every $\boldsymbol{A} \in \mathbf{A} \mathbf{L} \mathbf{G}^{*}(\mathrm{~L})$ holds: $\{\overline{1}\}$ is an $R_{1}$ L-filter iff $\{\overline{1}\}$ is an $R_{2}$-L-filter.

Again, taking for $R_{1}$ the 'fantastic property' and for $R_{2}$ the rule $\vdash((p \rightarrow \overline{0}) \rightarrow \overline{0}) \rightarrow p$ a simple syntactic proof (BL $+R_{2}$ is the usual presentation of Łukasiewicz logic) give us the second result from the introduction. In fact this theorem gives two ways of proving this kind of results: either syntactically or just by checking it for the filter $\{\overline{1}\}$.

In the next theorem we will cover the results of type three (supersets of special filters). We again assume that $\mathrm{L}+R$ is an axiomatic extension of $\mathrm{L}$. Thus there has to be some set of axioms $A x$ and we know that (due to the previous theorem) $F$ is $R$-Lfilter iff it is $A x$-L-filter. As elements of $A x$ are just axioms, this is equivalent to the fact that $e(\varphi) \in F$ for each $\varphi \in A x$. Clearly, this property is preserved if we take any $G \supseteq F$.

Theorem 16 (Results of type 3.) Let $R$ be $a$ set of $\mathcal{L}$-consecutions such that $\mathrm{L}+R$ is an axiomatic extension of $\mathrm{L}$, and $\boldsymbol{A} \in \mathbf{A} \mathbf{L} \mathbf{G}^{*}(\mathrm{~L})$. Further assume that $F$ is an $R$-L-filter on $\boldsymbol{A}$ and $G$ is an L-filter on $\boldsymbol{A}$ such that $F \subseteq G$.

Then $G$ is also an $R$-L-filter on $\boldsymbol{A}$.

Again, the particularization of this theorem for $\mathrm{BL}$ and fantastic filters gives us the third result from the introduction.

The final type of results deals with factorization by a filter $F$ (i.e., by the congruence $\sim_{F}$ ). We formulate the theorem in a more complex way, note that the equivalence of the the last three conditions is established in Theorem 14, and the equivalence of the first and the last claim can be proved in a straightforward way.

Theorem 17 (Results of type 4.) Let $R$ be a set of $\mathcal{L}$-consecutions such that $\mathrm{L}+R$ is an axiomatic extension of $\mathrm{L}, \boldsymbol{A} \in \mathbf{A} \mathbf{L G}^{*}(\mathrm{~L})$, and $F$ an $\mathrm{L}$-filter on $\boldsymbol{A}$. Then the following are equivalent: 
1. $F$ is an $R$-L-filter on $\boldsymbol{A}$,

2. $\boldsymbol{A} / F \in \mathbf{A} \mathbf{L} \mathbf{G}^{*}(\mathrm{~L}+R)$,

3. every L-filter on $\boldsymbol{A} / F$ is an $R$-L-filter,

4. $\{\overline{1}\}$ is $R$-L-filter on $\boldsymbol{A} / F$.

\section{Applications of general results}

Before we apply our results to derive particular examples known from the literature, we need to prove one crucial lemma. On the other hand, this lemma is all we need to start generating both known and previously unknown results.

Lemma 18 Using the name of the filter-defining property for the corresponding consecution (see Tables 4 and 5) we can prove:

- Classical logic is $\mathrm{FL}_{e w}$ (or any other logic from Table 2) + boolean or positive implicative or $(\neg \varphi \rightarrow \varphi) \rightarrow \varphi$.

- Classical logic is IMTL + implicative.

- Eukasiewicz logic is $\operatorname{divFL}_{e w}$ or $\mathrm{BL}+$ fantastic.

- Gödel logic is MTL or BL + implicative.

- Intuitionistic logic is $\mathrm{FL}_{e w}$ or $\operatorname{divFL}_{e w}+i m$ plicative.

Proof As an example, we are going to demonstrate the one but last statement: Let $R$ be the implicative condition. As MTL and BL both prove $\varphi \rightarrow(\varphi \rightarrow$ $\varphi \& \varphi)$ and $\varphi \rightarrow \varphi$ we obtain that both MTL $+R$ and $\mathrm{BL}+R$ proves $\varphi \rightarrow \varphi \& \varphi$, i.e., they extend the Gödel logic. Conversely, from the fact that $\varphi \rightarrow(\psi \rightarrow$ $\chi) \vdash \psi \rightarrow(\varphi \rightarrow \chi)$, holds in both MTL and BL we obtain $\varphi \rightarrow(\psi \rightarrow \chi), \varphi \rightarrow \psi \vdash \varphi \rightarrow(\varphi \rightarrow \chi)$, by transitivity. Residuation plus the Gödel axiom $\varphi \rightarrow \varphi \& \varphi$ completes the proof.

\subsection{Generation of known results}

Now we give a (small) sample of corollaries of our general results and the previous lemma together with reference to papers where it was proven in a 'direct' way.

Results of type 1

Corollary 19 ([7]) Let $\boldsymbol{A}$ be a BL-algebra. The following statements are equivalent.

1. Every BL-filter on $\boldsymbol{A}$ is an implicative/positive implicative/fantastic BL-filter.

2. $\{\overline{1}\}$ is an implicative/positive implicative/fantastic BL-filter on $\boldsymbol{A}$.

3. $\boldsymbol{A}$ is Gödel/Boolean/MV-algebra.

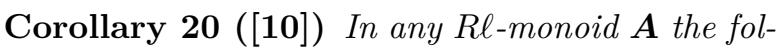
lowing conditions are equivalent:

1. Every filter on $\boldsymbol{A}$ is an implicative/positive implicative/fantastic filter.

2. $\{\overline{1}\}$ is an implicative/positive implicative/fantastic filter.
3. $\boldsymbol{A}$ is Heyting algebra/Boolean/MV-algebra.

Corollary 21 ([12]) In any residuated lattice $\mathbf{L}$ the following conditions are equivalent:

1. Every filter on $\mathbf{L}$ is a Boolean/regular filter.

2. $\{\overline{1}\}$ is a Boolean/regular filter.

3. $\mathbf{L}$ is Boolean algebra/involutive residuated lattice i.e. residuated lattice satisfying $\neg \neg x=x$ for all $x \in \mathbf{L}$.

Results of type 2.

Corollary 22 ([9]) For any filter $F$ of a BLalgebra $\mathbf{A}$, the following conditions are equivalent:

1. $F$ is an implicative/positive implicative/fantastic filter,

2. $x \rightarrow x \& x \in F /(\neg x \rightarrow x) \rightarrow x \in F /$ $\neg \neg x \rightarrow x \in F$ for every $x \in A$.

Corollary 23 ([9], [10]) Boolean and positive implicative filters of any BL-algebra coincide.

Corollary 24 ([10]) If $F$ is a filter of an $R \ell$ monoid $\boldsymbol{A}$, then the following conditions are equivalent:

1. F is a fantastic filter of $\boldsymbol{A}$.

2. $\neg \neg x \rightarrow x \in F$ for every $x \in A$.

Results of type 3.

Corollary 25 ([7]) Let $\boldsymbol{A}$ be a BL-algebra. If $F$ is a implicative/positive implicative/fantastic filter on $\boldsymbol{A}$, then every filter $G$ containing $F$ is also an implicative/positive implicative/fantastic filter.

Corollary 26 ([10]) If $F$ and $G$ are filters of

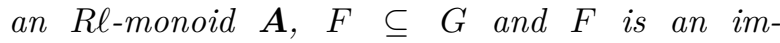
plicative/positive implicative/fantastic filter on $\boldsymbol{A}$, then $G$ is also an implicative/positive implicative/fantastic filter on $\boldsymbol{A}$.

Corollary 27 ([12]) Let $\boldsymbol{L}$ be a residuated lattice. If $F$ is a regular filter of $\boldsymbol{L}$, then every filter $G$ containing $F$ is also a regular filter of $\boldsymbol{L}$.

Results of type 4.

Corollary 28 ([7]) Let $\boldsymbol{A}$ a BL-algebra, $F$ a filter. Then $F$ is an implicative/positive implicative/fantastic filter on $\boldsymbol{A}$ if and only if every filter on the quotient algebra $\boldsymbol{A} / F$ is an implicative/positive implicative/fantastic filter.

Corollary 29 ([10]) A filter $F$ of an R $\ell$-monoid $\boldsymbol{A}$ is Boolean/implicative/fantastic if and only if $\boldsymbol{A} / F$ is an Boolean/Heyting/MV-algebra.

Corollary 30 ([12]) Let $F$ be a filter of a residuated lattice $\boldsymbol{L}$. Then the following assertions are equivalent:

1. F is a Boolean filter of $\boldsymbol{L}$.

2. The quotient residuated lattice $\boldsymbol{L} / F$ is a Boolean algebra. 


\subsection{Generating new 'original' results}

Now we are going to demonstrate how our theory could be (ab)used to generate new 'original' results matching presented patterns. We can formulate results that (probably) nobody formulated so far. First, we could generalize known results to other classes of algebras:

Corollary 31 (Type 1.) Let $\boldsymbol{A}$ be an IMTLalgebra. The following statements are equivalent.

1. Every IMTL-filter on $\boldsymbol{A}$ is an implicative IMTL-filter.

2. $\{\overline{1}\}$ is an implicative IMTL-filter on $\boldsymbol{A}$.

3. $\boldsymbol{A}$ is Boolean algebra.

Corollary 32 (Type 2.) Boolean and implicative filters of any IMTL-algebra coincide.

Corollary 33 (Type 3.) Let $\boldsymbol{A}$ be an IMTLalgebra. If $F$ is a implicative filter, then every filter $G$ containing $F$ is also a implicative.

Corollary 34 (Type 4.) A filter $F$ of an IMTLalgebra $\boldsymbol{A}$ is implicative iff every filter on the quotient algebra $\boldsymbol{A} / F$ is implicative.

Second, we could also define new classes of filters (with as good motivation as those defined in the literature have) and prove all four types of results.

Definition 35 Let $\boldsymbol{A}$ be a MTL-algebra. A filter $F$ is called a contractional filter on $\boldsymbol{A}$ if for all $x \in A$ we have $x \rightarrow y \in F$ whenever $x \rightarrow(x \rightarrow y) \in F$.

As we know that MTL + contrational property (expressed as consecution) is Gödel logic, we immediately obtain:

Corollary 36 (Type 1.) In any MTL-algebra $\boldsymbol{A}$ the following conditions are equivalent:

1. Every filter on $\boldsymbol{A}$ is a contractional filter.

2. $\{\overline{1}\}$ is a contractional filter.

3. $\boldsymbol{A}$ is a Gödel algebra.

Corollary 37 (Type 2.) Contractional and implicative filters of any MTL-algebra coincide.

Corollary 38 (Type 3.) Let $\boldsymbol{A}$ be an MTLalgebra, $F, G \subseteq A$ filters. If $F \subseteq G$ and $F$ is a contractional filter, then $G$ is also a contractional filter.

Corollary 39 (Type 4.) Let $\boldsymbol{A}$ a MTL-algebra and $F$ an MTL-filter. Then $F$ is an contractional filter on $\boldsymbol{A}$ if and only if every MTL-filter on the quotient algebra $\boldsymbol{A} / F$ is a contractional filter.

\section{Acknowledgments}

The work of Martin Víta was supported by grant GD401/09/H007 of the Grant Agency of the Czech Republic.

The work of Petr Cintula was supported partly by project $1 \mathrm{M} 0545$ of the Ministry of Education, Youth, and Sports of CR and party by Institutional Research Plan AVOZ10300504.

\section{References}

[1] Willem J. Blok and Don Pigozzi. Algebraizable Logics, volume 396 of Memoirs of the American Mathematical Society. American Mathematical Society, Providence, RI, 1989.

[2] Petr Cintula and Carles Noguera. Implicational (semilinear) logics I: Basic notions and hierarchy. Archive for Mathematical Logic, 49(4):417-446, 2010.

[3] Janusz Czelakowski. Protoalgebraic Logics, volume 10 of Trends in Logic. Kluwer, Dordrecht, 2001.

[4] Josep Maria Font, Ramon Jansana, and Don Pigozzi. A survey of Abstract Algebraic Logic. Studia Logica, 74(1-2, Special Issue on Abstract Algebraic Logic II):13-97, 2003.

[5] Nikolaos Galatos, Peter Jipsen, Tomasz Kowalski, and Hiroakira Ono. Residuated Lattices: An Algebraic Glimpse at Substructural Logics, volume 151 of Studies in Logic and the Foundations of Mathematics. Elsevier, Amsterdam, 2007.

[6] Petr Hájek. Metamathematics of Fuzzy Logic, volume 4 of Trends in Logic. Kluwer, Dordrecht, 1998.

[7] M. Haveshki, A. Saeid, and E. Eslami. Some types of filters in BL-algebras. Soft Computing, 10:657-664, 2006.

[8] Ulrich Höhle. Commutative, residuated lmonoids. In Ulrich Höhle and Erich Peter Klement, editors, Non-Classical Logics and Their Applications to Fuzzy Subsets, pages 53-106. Kluwer, Dordrecht, 1995.

[9] M. Kondo and W. A. Dudek. Filter theory of BL-algebras. Soft Computing, 12:419-423, 2008.

[10] Jiří Rachůnek and Dana Šalounová. Classes of filters in generalizations of commutative fuzzy structures. Acta Universitatis Palackianae Olomucensis. Facultas Rerum Naturalium. Mathematica, 48(1):93-107, 2009.

[11] Helena Rasiowa. An Algebraic Approach to Non-Classical Logics. North-Holland, Amsterdam, 1974.

[12] Y. Zhu and Y. Xu. On filter theory of residuated lattices. Information Sciences, 180(19):3614-3632, 2010. 